\title{
Evidence-based sizing of non-inferiority trials using decision models
}

\author{
Iris Lansdorp-Vogelaar ${ }^{1}$, Reshma Jagsi ${ }^{2}$, Jinani Jayasekera ${ }^{3}$, Natasha K. Stout ${ }^{4}$, Sandra A. Mitchell ${ }^{5}$ and \\ Eric J. Feuer ${ }^{6 *}$ (i)
}

\begin{abstract}
Background: There are significant challenges to the successful conduct of non-inferiority trials because they require large numbers to demonstrate that an alternative intervention is "not too much worse" than the standard. In this paper, we present a novel strategy for designing non-inferiority trials using an approach for determining the appropriate non-inferiority margin $(\delta)$, which explicitly balances the benefits of interventions in the two arms of the study (e.g. lower recurrence rate or better survival) with the burden of interventions (e.g. toxicity, pain), and early and late-term morbidity.

Methods: We use a decision analytic approach to simulate a trial using a fixed value for the trial outcome of interest (e.g. cancer incidence or recurrence) under the standard intervention $\left(p_{S}\right)$ and systematically varying the incidence of the outcome in the alternative intervention $\left(p_{A}\right)$. The non-inferiority margin, $p_{A}-p_{S}=\delta$, is reached when the lower event rate of the standard therapy counterbalances the higher event rate but improved morbidity burden of the alternative. We consider the appropriate non-inferiority margin as the tipping point at which the quality-adjusted life-years saved in the two arms are equal.
\end{abstract}

Results: Using the European Polyp Surveillance non-inferiority trial as an example, our decision analytic approach suggests an appropriate non-inferiority margin, defined here as the difference between the two study arms in the 10 -year risk of being diagnosed with colorectal cancer, of $0.42 \%$ rather than the $0.50 \%$ used to design the trial. The size of the non-inferiority margin was smaller for higher assumed burden of colonoscopies.

Conclusions: The example demonstrates that applying our proposed method appears feasible in real-world settings and offers the benefits of more explicit and rigorous quantification of the various considerations relevant for determining a non-inferiority margin and associated trial sample size.

Keywords: Non-inferiority trial, Sample size, Power calculation, Quality-adjusted life years, Non-inferiority margin

\section{Background}

Traditionally, trials investigating de-escalation of interventions have employed a non-inferiority design, requiring large numbers to convincingly demonstrate that an alternative treatment is "not too much worse" than a standard treatment with known benefit. This definition of benefit conventionally focuses on disease control and/ or prevention outcomes alone, without explicit quantification or consideration of the differential impact of

\footnotetext{
* Correspondence: rf41u@nih.gov

${ }^{6}$ Statistical Research and Applications Branch, Surveillance Research Program, Division of Cancer Control and Population Sciences, National Cancer Institute, 9609 Medical Center Drive, Room 4E534, Bethesda, MD 20892-9765, USA

Full list of author information is available at the end of the article
}

different regimens on toxicity, burden, and early and late-term morbidity.

Determination of the non-inferiority margin is the most critical step in non-inferiority testing, as it represents the point of equipoise where the benefits of the standard therapy compared to the lesser alternative are outweighed by its risks or perhaps its additional costs [1].

While there are several guidelines available to aid researchers in the development of non-inferiority margins in these trial designs [2-9], a recent systematic review of non-inferiority trials showed that the majority of previously published trials have either provided ambiguous or limited information to justify their choice of margin [10]. Lack of robust justifications for setting non-inferiority

(c) The Author(s). 2019 Open Access This article is distributed under the terms of the Creative Commons Attribution 4.0 International License (http://creativecommons.org/licenses/by/4.0/), which permits unrestricted use, distribution, and reproduction in any medium, provided you give appropriate credit to the original author(s) and the source, provide a link to the Creative Commons license, and indicate if changes were made. The Creative Commons Public Domain Dedication waiver (http://creativecommons.org/publicdomain/zero/1.0/) applies to the data made available in this article, unless otherwise stated. 
margins in standard practice could lead to inconsistency in recommendations and guidelines based on non-inferio rity trials. More importantly, current guidelines do not provide strict standards for considering risks, morbidity and costs in the determination of non-inferiority margins, necessitating large clinical trials to evaluate these sorts of questions.

In this paper, we introduce a general decision analytic framework that uses an explicit net-benefit approach for determination of non-inferiority margins for optimal design of non-inferiority trials in oncology as well as other disease settings. In this context, quality-adjusted life years (QALYs) can be used as a single integrated measure of health outcomes that represent both the quality and quantity of life lived [11]. If QALY loss of a current intervention compared to an alternative are substantial, a larger cancer-control benefit would be necessary to justify its continued use.

In this paper, we introduce a novel methodology based on decision-modeling for setting the non-inferiority margin of trials investigating de-escalation of interventions. Examples of such trials are those that investigate: 1 ) omitting (parts of) the treatment regimen, 2) lower drug-dose regimens, 3) alternative drugs with less side effects and 4) lower frequency of follow-up exams, In the next section, we briefly describe the general concepts behind the methodology before illustrating its applicability.

\section{Methods}

\section{Non-inferiority trials and the non-inferiority margin}

The traditional approach to non-inferiority trials in oncology tests whether a new experimental treatment is not meaningfully worse than an existing treatment in terms of a disease outcome (e.g. cancer recurrence rate) [12]. The concept of "meaningfully worse" is formalized in the definition of a value called the non-inferiority margin, or more generally the equivalence margin, denoted by $\delta$. The non-inferiority margin defines the maximum clinically acceptable difference that one is willing to accept in return for the lower burden, morbidity and/or costs of the new therapy [1]. Non-inferiority trials have a null hypothesis that the alternative treatment is inferior to the standard treatment by at least the pre-specified non-inferiority margin $\left(\mathrm{Ho}: \mathrm{p}_{\mathrm{A}}-\mathrm{p}_{\mathrm{S}}>\delta\right.$, where $\mathrm{p}_{\mathrm{S}}$ and $\mathrm{p}_{\mathrm{A}}$ are event rates of the outcome of interest for standard and alternative interventions, respectively). The alternative hypothesis is that the alternative treatment is not inferior to the standard treatment (i.e., is less than the non-inferiority margin (Ha: $\left.\mathrm{p}_{\mathrm{A}}-\mathrm{p}_{\mathrm{S}}<\delta\right)$ ).

Several methods have been proposed for setting the non-inferiority margin. Two methods, the 95-95 method and the synthesis method, are prescribed by the FDA in the evaluation of new interventions to ensure that the non-inferiority margin does not overlap the event rate in a control population [9]. Neither of these methods account for the difference in morbidity outcomes between the interventions, so even if the new intervention is better than the placebo in terms of mortality or event rates, the margin does not guarantee that the new intervention is truly non-inferior to the current intervention. Thus, while these methods are useful to ensure that the new intervention is superior to a control, it does not obviate the need to still quantify the non-inferiority margin in terms of the harms and benefits of the standard and new intervention. The methods can therefore be considered additional constraints.

The Delphic method does take morbidity into account, as it asks physicians or patients to subjectively assess how much benefit they might forgo to avoid the potential incremental harms of the standard therapy [12]. However, it may not do so in a reproducible, systematic way, especially with respect to the long-term implications of the interventions being compared. Setting a framework for an evidence-based quantification of the non-inferiority margin is therefore the focus of this paper.

\section{Proposed decision analytic approach}

Our proposed framework utilizes a decision analytic approach to simulate a trial using a fixed value for cancer incidence or recurrence rates under the standard intervention $\left(\mathrm{p}_{\mathrm{s}}\right)$ and systematically varying cancer incidencelrecurrence under the alternative intervention $\left(\mathrm{p}_{\mathrm{A}}\right)$. The non-inferiority margin, $\mathrm{p}_{\mathrm{A}}-\mathrm{p}_{\mathrm{S}}=\delta$, is reached when the lower cancer incidence or recurrence rate of the standard therapy is counterbalanced by the higher disease rate but improved morbidity burden of the alternative. This is quantified in a decision model as the level of incidence/recurrence $\left(\mathrm{p}_{\mathrm{A}}\right)$ at which the QALYs in the two arms are equal. Therefore, true non-inferiority, as operationalized in this paper, is established when the potential loss in life-years due to lower efficacy in the alternative intervention is offset by an increase in quality of life from lower burden and/or side effects relative to the standard intervention. While some trials have used outcome measures of net benefit, this paper advances the literature by using decision analytic models and measures of net benefit to explicitly quantify the non-inferiority margin, and, by extension, formally size the trial.

Our proposed framework consists of four steps (Fig. 1), which are illustrated with an example in the next section. In Step 1, a decision model is formulated as a framework for quantifying the lifelong impacts of outcomes by integrating the probabilities of specific outcomes and their sequalae with their disutilities. An existing decision model can be used, or one can be developed de novo. Disutilities can be obtained from previous studies or literature. A model can be simple, including only the relevant types of 


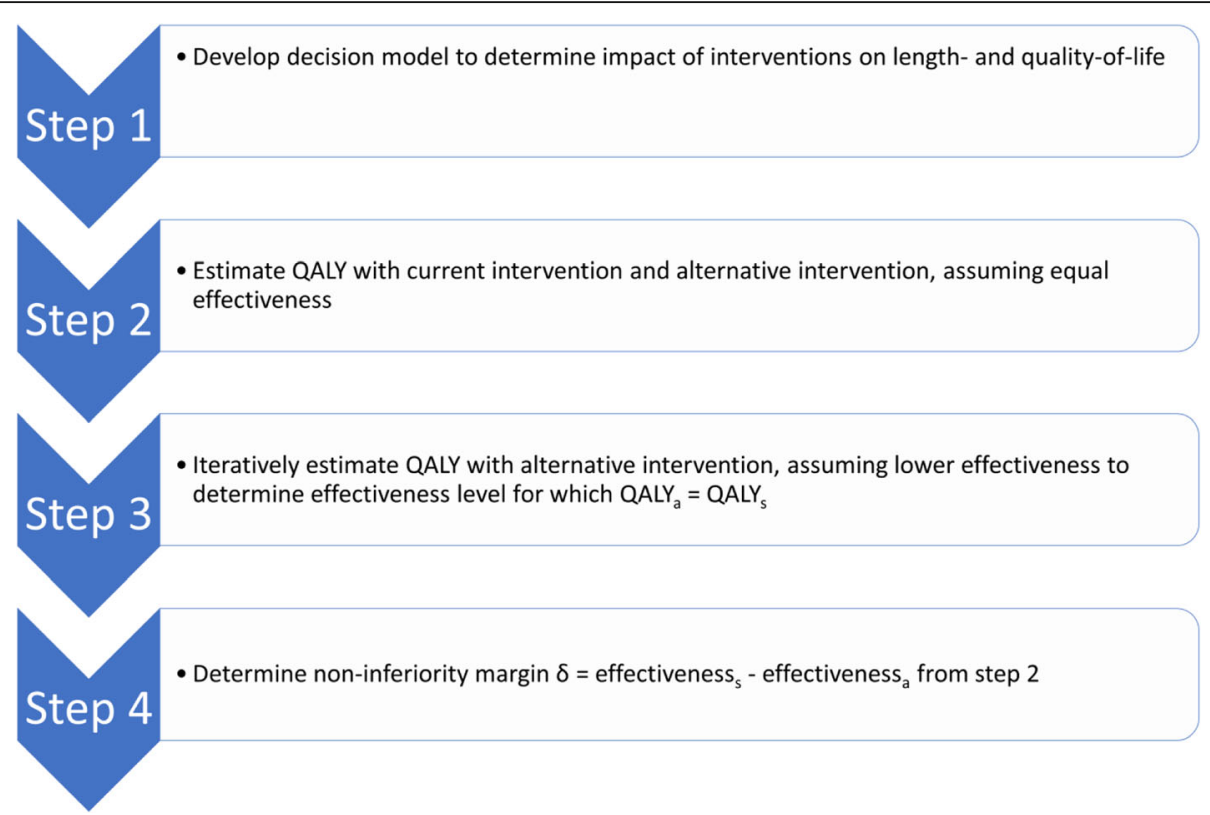

Fig. 1 Overview of four steps of proposed methodology to determine evidence-based non-inferiority margin in non-inferiority trials. " $s$ " represents the standard intervention and "a" represents the alternative scaled back intervention

adverse events and their associated disutilities post cancer diagnosis over the remaining life course.

In Step 2 we use the decision model to estimate how the alternative intervention would improve the quality of life of the patient, if it would have the same effectiveness as the current intervention.

In Step 3, the model is used to estimate quality of life under the assumption that the alternative intervention is slightly less effective than the standard intervention, and therefore may result in either more deaths or earlier death with an associated decrease in length of life. A less effective alternative intervention may also result in additional incident or recurrent cases of the disease and thus a loss in quality of life on top of the loss in average length of life. By iteratively evaluating the effectiveness of the alternative intervention, we can find the point at which the QALYs lost from the lower effectiveness is equal to the QALYs gained from the lower burden and/ or side effect of the alternative intervention. Finally, in Step 4, the difference in effectiveness between the current intervention and the derived effectiveness level for the alternative intervention can then be used as the non-inferiority margin for evidence-based sizing of non-inferiority trials.

\section{Alternative measures for non-inferiority}

Using disutilities from literature for determining equal QALYs implies that a current intervention may be replaced by an alternative when it is non-inferior for those whose utility weights do not diverge dramatically from the average member of the population. A more conservative approach would be to extend the trial applicability to a larger fraction of the population, which would require down weighting the disutility associated with the toxicity or burden of the intervention relative to the average population, leading to a smaller non-inferiority margin and therefore a larger sample size. Analyses along these lines are possible and would be similar to the one described above, but instead of using average disutilities, they might consider utility weights derived from a different cut-point of the population distribution (e.g., the 25th percentile).

Alternative interventions or omission of an intervention may not just be less harmful but may also be less expensive for the target population. As such, a non-inferiority margin could also be designed if lower costs could make up for a certain reduction in effectiveness. The decision analytic method is largely the same as above, except that the margin is determined based on cost-effectiveness (costs per QALY) rather than QALY. The non-inferiority margin is established based on the level of effectiveness such that the reduction in costs no longer compensates enough for the reduction in QALYs given a willingness-to-pay threshold (e.g., the ratio is at or above a threshold).

\section{Uncertainty analysis}

Some parameters assumed to be known in the model, in fact are not known with certainty. To account for this uncertainty, one can repeat the proposed approach for different parameter values. This way, insights are obtained on the sensitivity of the power calculation to different model assumptions. To ensure sufficient power, 
one could consider using a sample size in the upper ranges of the uncertainty analysis.

\section{Results}

We chose the European Polyp Surveillance (EPoS) study [13] as an example of a non-inferiority trial to illustrate how this decision-analytic approach can help inform the non-inferiority margin and design of non-inferiority trials. We used the MISCAN-Colon microsimulation model as the decision analytic model to estimate QALYs and costs for this example [14]. We first briefly describe the trial and the model before presenting results with respect to applying the framework to establishing an evidence-based non-inferiority margin.

\section{EPoS trial}

The EPoS study consists of two ongoing randomized controlled trials and a planned cohort follow-up study. A detailed description of the design of the trial was recently published [13]. In this example, we focus on the EPoS I randomized controlled trial. In brief, in EPoS I, low-risk adenoma patients (i.e., patients with 1-2 small tubular adenomas without high-grade dysplasia or villousness) were randomized to receive surveillance colonoscopy at both years 5 and 10 (standard intervention) vs. surveillance colonoscopy at 10 years only (suggested alternative scaled-back intervention).

The study was powered as a non-inferiority trial, since the investigators wanted to determine if the 10-year colorectal cancer (CRC) incidence for the scaled-back intervention fell within a specified margin of that previously observed for the standard intervention. The expected 10-year CRC incidence under the standard intervention is approximately $1 \%$. For the power calculation, it was felt that a 10-year CRC incidence rate of up to $1.5 \%$ could be tolerated to gain the advantages of having surveillance colonoscopies half as frequently. Thus, a non-inferiority margin of $0.5 \%$ was used. Based on $90 \%$ power and a one-sided alpha of 0.05 , it was estimated that a total of 13,766 individuals needed to be included in EPoS I.

\section{MISCAN-Colon}

MISCAN-Colon is a well-established microsimulation model for CRC developed at the Department of Public Health of the Erasmus University Medical Center (Rotterdam, the Netherlands) $[15,16]$. It is one of the models participating in the National Cancer Institute's Cancer Intervention and Surveillance Modeling Network (CISNET) [17-19]. The model's structure, underlying assumptions, and calibration are described in previous publications $[15,20]$. Briefly, the model simulates the life histories of individuals from birth to death. CRC arises in the population according to the adenoma-carcinoma sequence. Screening and surveillance may alter these life histories through possible removal of adenomas and detection of cancers. In this way CRC mortality can be reduced. The life years gained by screening are calculated as the difference in model-predicted life years lived in the population with and without CRC screening.

We used MISCAN-Colon to simulate the EPoS I study population of individuals diagnosed with low-risk adenomas and undergoing subsequent surveillance. We simulated two colonoscopy surveillance strategies, one with surveillance every 5 years, and one with surveillance every 10 years. Surveillance was assumed to continue until age 75 . We followed individuals for their lifetimes. The model was used to predict lifetime QALYs and costs of the standard and alternative surveillance interventions. Assumptions for natural history and costs were based on previous work [21].

\section{Disutilities from surveillance colonoscopy and CRC}

The potential gain in QALYs from the less intensive surveillance interval compared to the more intensive interval stems from the reduction in colonoscopies required, as colonoscopies are associated with patient burden and complications. For this analysis, disutilities were chosen by assumption. We assumed a population-average disutility of 3.1 days for every colonoscopy performed to account for 3 weeks of anxiety prior to colonoscopy at a disutility of 0.1 , and 2 days of preparation and procedure at a disutility of 0.5 . In addition, we modeled age-specific risks for gastrointestinal and cardiovascular complications of colonoscopy. The overall risk associated with colonoscopies with polypectomy increased exponentially with age: from 2 complications per 1000 colonoscopies at age 40 to 38 per 1000 at age 85 [15]. Colonoscopies without polypectomy were not associated with a risk for complications. We assumed a utility loss of two weeks of life per complication from colonoscopy. We assumed that one out of every 30,000 colonoscopies involving polypectomy resulted in death.

On the other hand, treatment for CRC is also associated with a loss in quality of life, and higher rates of recurrence may therefore have a negative impact on QALYs. Disutilities for life-years with CRC were therefore also incorporated, based on findings by Ness et al [22].

\section{Determining the non-inferiority margin Estimate QALYs with less intensive surveillance assuming equal effectiveness}

Assuming a $1.0 \%$ risk of CRC incidence with the standard intervention of 5-yearly surveillance, the model predicted 22,424 life-years per 1000 50-year old adenoma patients. Per 1000 adenoma patients, 26.3 QALYs would be lost due to surveillance colonoscopies and another 0.3 QALYs 
Table 1 Comparison of QALY outcomes across various scenarios of surveillance for adenoma patients and CRC risk

\begin{tabular}{|c|c|c|c|c|c|c|c|}
\hline Strategy & $\begin{array}{l}10 y \\
\text { cumulative } \\
\text { incidence }\end{array}$ & $\begin{array}{l}\text { Life-years } \\
\text { (per } 1000 \\
\text { pt) }\end{array}$ & $\begin{array}{l}\text { QALYs lost due to } \\
\text { surveillance (per } 1000 \\
\text { pt) }\end{array}$ & $\begin{array}{l}\text { QALYs lost due to } \\
\text { complications (per } 1000 \\
\text { pt) }\end{array}$ & $\begin{array}{l}\text { QALYs lost due to } \\
\text { treatment (per } 1000 \\
\text { pt) }\end{array}$ & $\begin{array}{l}\text { QALYS } \\
\text { (per } 1000 \\
\text { pt) }\end{array}$ & $\begin{array}{l}\text { Difference in } \\
\text { QALYs (per } 1000 \\
\text { pt) }\end{array}$ \\
\hline $\begin{array}{l}\text { 5-yearly } \\
\text { surveillance }\end{array}$ & $1.0 \%$ & 22,424 & 26.3 & 0.3 & 54.1 & $22,343.2$ & $\begin{array}{l}\text { Reference } \\
\text { Strategy }\end{array}$ \\
\hline $\begin{array}{l}\text { 10-yearly } \\
\text { surveillance }\end{array}$ & $1.0 \%$ & 22,424 & 18.0 & 0.2 & 54.1 & $22,351.5$ & 8.3 \\
\hline $\begin{array}{l}\text { 10-yearly } \\
\text { surveillance }\end{array}$ & $1.5 \%$ & 22,412 & 18.1 & 0.2 & 55.8 & $22,338.1$ & -5.1 \\
\hline $\begin{array}{l}\text { 10-yearly } \\
\text { surveillance }\end{array}$ & $1.3 \%$ & 22,419 & 18.0 & 0.2 & $49.8^{\mathrm{a}}$ & $22,351.0$ & 8.1 \\
\hline $\begin{array}{l}\text { 10-yearly } \\
\text { surveillance }\end{array}$ & $1.42 \%$ & 22,415 & 18.1 & 0.2 & $53.5^{\mathrm{a}}$ & $22,343.1$ & 0.0 \\
\hline
\end{tabular}

10y: 10-year; QALYs: Quality-adjusted life-years; pt.: patients

${ }^{a}$ QALY lost to treatment are not necessarily higher with higher CRC incidence, because they also depend on the stage distribution of CRC cases and time spent in each CRC state. Higher CRC incidence may lead to fewer life-years with CRC treatment because of higher mortality from CRC

due to complications of surveillance (Table 1). In addition, 54.1 QALYs per 1000 would be lost due to diagnosis and treatment of CRC. Together, this resulted in total of 22,343 QALYs per 1000 adenoma patients (Table 1).

Under an initial assumption of equal effectiveness (a $1.0 \%$ risk of CRC incidence) for both 10 -yearly and 5 -yearly surveillance, life-years with the alternative surveillance schedule of 10 -yearly surveillance were the same as with 5 -yearly surveillance, i.e., 22,424 years per 1000 adenoma patients. However, because of a reduction in colonoscopies performed, the QALYs lost to surveillance are lower (i.e., 18.1 and 0.2 for colonoscopies and complications, respectively). Consequently, QALYs for the alternative intervention were higher at 22,352 years. This amounts to an improvement in QALYs of 8.3 per
1000 adenoma patients compared to the standard intervention.

\section{Iteratively determine level of effectiveness for equal QALYs}

For a $1.5 \%$ risk of CRC incidence at 10 years under 10-yearly surveillance, which was used to design EPoS I, QALYs would be 22,338 years per 1000 adenoma patients. This is slightly lower than the QALYs from the current intervention (Fig. 2). If 10-yearly surveillance would lead to a 10 -year risk of CRC incidence of $1.3 \%$, total QALYs would be 22,351 years, for a gain in QALYs of 7.8 years per 1000 adenoma patients (Table 1). Through iterative running of the MISCAN-Colon model for different levels of 10-year risks of CRC incidence in adenoma patients with 10 -yearly surveillance, we found

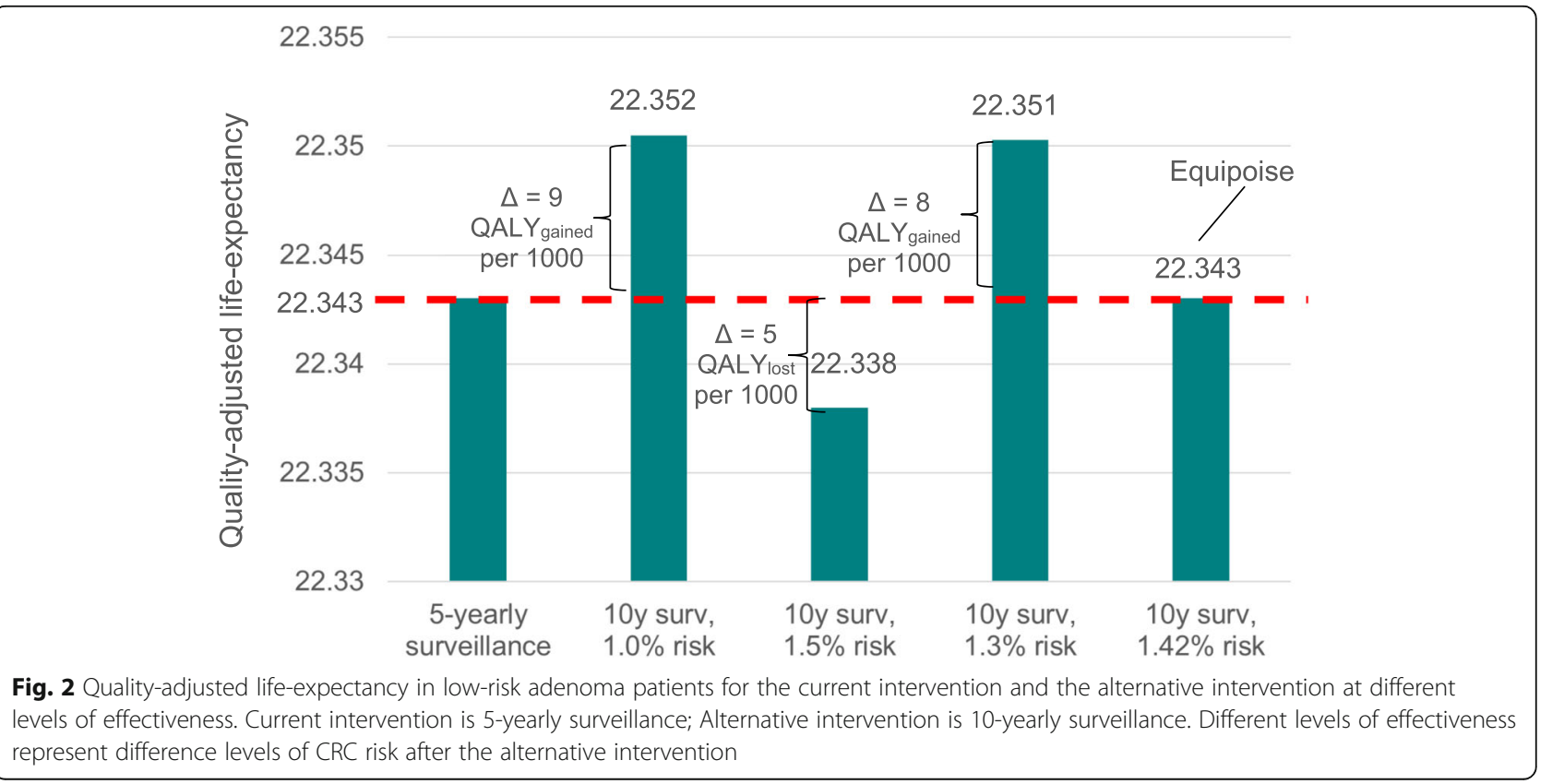


Table 2 Non-inferiority margins and sample size requirements for 5-yearly surveillance (vs. 10-yearly surveillance) of low-risk adenoma patients

\begin{tabular}{|c|c|c|c|c|c|c|c|}
\hline Strategy & $\begin{array}{l}10 y \\
\text { cumulative } \\
\text { incidence }\end{array}$ & $\begin{array}{l}\text { Life-years } \\
\text { (per } 1000 \\
\text { pt) }\end{array}$ & $\begin{array}{l}\text { QALYs lost to } \\
\text { surveillance (per } \\
1000 \mathrm{pt})\end{array}$ & $\begin{array}{l}\text { QALYs lost to surveillance } \\
\text { complications (per } 1000 \text { pt) }\end{array}$ & $\begin{array}{l}\text { QALYs lost to CRC } \\
\text { treatment (per } 1000 \\
\text { pt) }\end{array}$ & $\begin{array}{l}\text { QALYs } \\
\text { (per } 1000 \\
\text { pt) }\end{array}$ & $\begin{array}{l}\text { Required } \\
\text { sample size }\end{array}$ \\
\hline \multicolumn{8}{|c|}{ Non-inferiority based on effectiveness and average disutilities (base case) - 3.1 days lost per colonoscopy } \\
\hline $\begin{array}{l}\text { 5-yearly } \\
\text { surveillance }\end{array}$ & $1.0 \%$ & 22,424 & 26.3 & 0.3 & 54.1 & 22,343 & 19,234 \\
\hline $\begin{array}{l}\text { 10-yearly } \\
\text { surveillance }\end{array}$ & $1.42 \%$ & 22,415 & 18.1 & 0.2 & 53.5 & 22,343 & \\
\hline \multicolumn{8}{|c|}{ Non-inferiority based on cost-effectiveness using threshold of €20,000 per QALY gained and base case disutilities (3.1 days lost per colonoscopy) } \\
\hline $\begin{array}{l}5 \text {-yearly } \\
\text { surveillance }\end{array}$ & $1.0 \%$ & 22,424 & 26.3 & 0.3 & 54.1 & 22,343 & 8826 \\
\hline $\begin{array}{l}\text { 10-yearly } \\
\text { surveillance }\end{array}$ & $1.62 \%$ & 22,408 & 18.2 & 0.2 & 59.2 & 22,331 & \\
\hline \multicolumn{8}{|c|}{ Non-inferiority based on $80 \%$ of base disutilities ( 2.5 days lost per colonoscopy) } \\
\hline $\begin{array}{l}5 \text {-yearly } \\
\text { surveillance }\end{array}$ & $1.0 \%$ & 22,424 & 21.0 & 0.3 & 54.1 & 22,348 & 21,206 \\
\hline $\begin{array}{l}\text { 10-yearly } \\
\text { surveillance }\end{array}$ & $1.40 \%$ & 22,416 & 14.5 & 0.2 & 52.8 & 22,348 & \\
\hline \multicolumn{8}{|c|}{ Non-inferiority based on $120 \%$ of base disutilties ( 3.7 days lost per colonoscopy) } \\
\hline $\begin{array}{l}\text { 5-yearly } \\
\text { surveillance }\end{array}$ & $1.0 \%$ & 22,424 & 31.5 & 0.3 & 54.1 & 22,338 & 16,754 \\
\hline $\begin{array}{l}\text { 10-yearly } \\
\text { surveillance }\end{array}$ & $1.45 \%$ & 22,414 & 21.7 & 0.2 & 54.2 & 22,338 & \\
\hline
\end{tabular}

that at a risk of CRC incidence of $1.42 \%$, QALYs from 5 -yearly surveillance and 10-yearly surveillance would be equal (Table 2).

\section{Calculate the non-inferiority margin}

To demonstrate non-inferiority of 10-yearly surveillance compared to 5-yearly surveillance in low-risk adenoma patients, a non-inferiority margin of $0.42 \%$ (1.42-1\%) should be used in the power calculations. Assuming this non-inferiority margin and an expected CRC risk of $1.0 \%$ with the standard intervention, 9617 individuals need to be included in each arm of the trial for a total sample size of 19,234 adenoma patients (Table 2).

\section{Alternative non-inferiority margins}

Assuming a lower disutility for being in colonoscopy surveillance (disutility of 2.5 days or $80 \%$ of base-case value) to correspond with a larger fraction of the population, QALYs were equal at a lower 10-year CRC risk for the 10-yearly surveillance arm of $1.4 \%$ (Table 2). The associated sample size with this non-inferiority margin of $0.4 \%$ would be 21,206 adenoma patients. Alternatively, assuming a higher disutility for being in colonoscopy surveillance (disutility of 3.7 days or $120 \%$ of base-case value) yields a non-inferiority margin of $0.45 \%$ and a corresponding sample size of 16,754 adenoma patients (Table 22).

Basing the non-inferiority margin for EPoS I on cost-benefit with a standard Dutch threshold of $€ 20,000$ per life year gained, rather than net benefit alone, would lead to a higher CRC incidence risk of $1.62 \%$ allowed in the non-standard arm for non-inferiority (Table 2). With this non-inferiority margin of $0.62 \%$, the required sample size for $90 \%$ power would be 8826 adenoma patients (4413 per arm).

\section{Uncertainty analysis on disutility and colonoscopy sensitivity}

Figure 3 shows the line of equipoise as a function of the self-perceived disutiities of colonoscopy. The figure shows the translation between disutility of colonoscopy and the resulting QALY gained from 5-yearly surveillance, as well as the associated non-inferiority margin and required sample size to demonstrate non-inferiority. In general, lower assumptions for disutilities associated with colonoscopy resulted in proportionally lower levels of $\mathrm{CRC}$ incidence risk allowable in the non-standard arm for equal QALYs for 5- and 10-yearly surveillance. Figure 4 shows the same results in addition to results when assuming 5\% higher and 5\% lower estimates for colonoscopy sensitivity. Figure 4 clearly demonstrates the sensitivity of required sample sizes for assumptions 


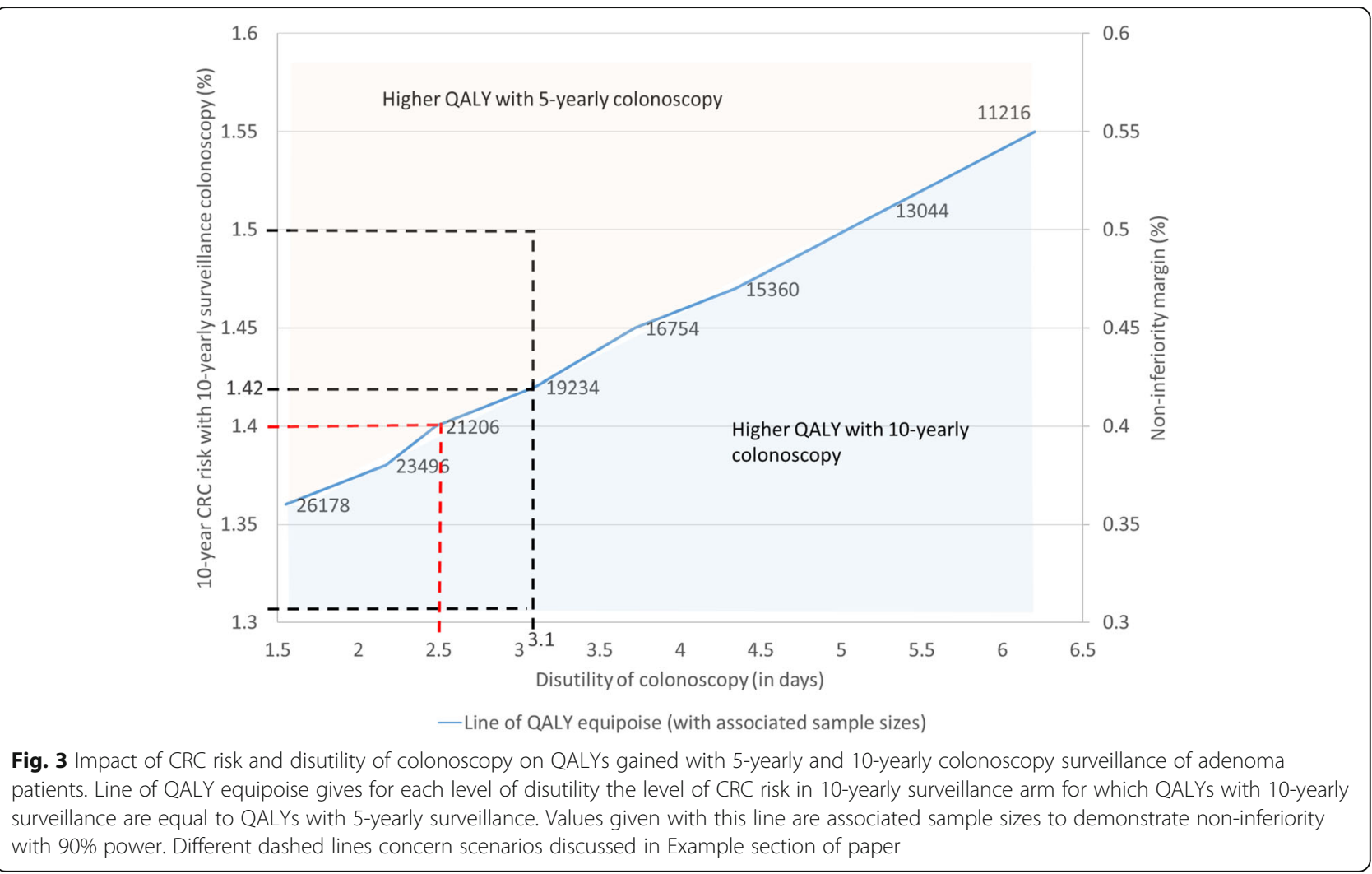

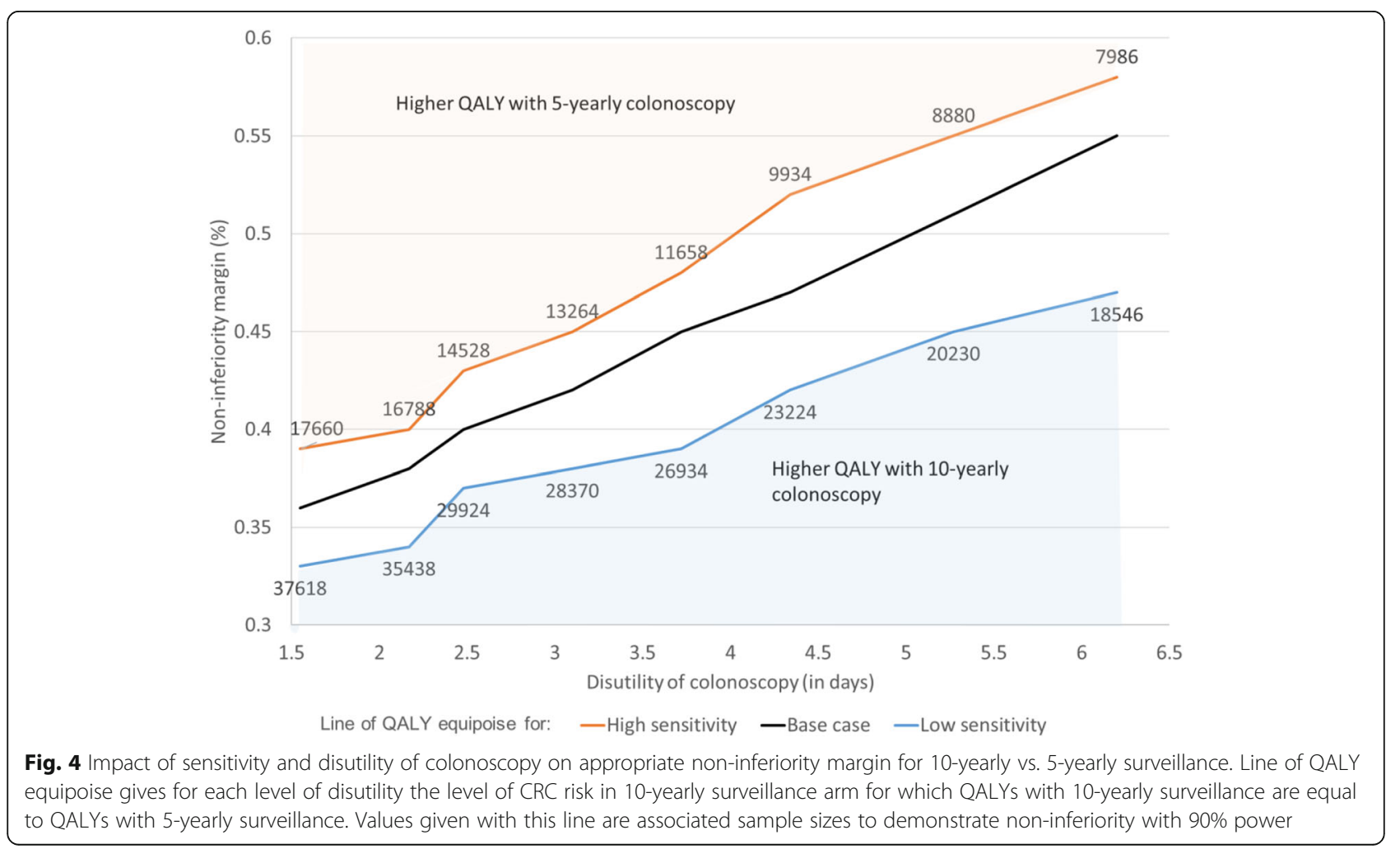


about colonoscopy sensitivity and disutility One could consider using a sample size of around 30,000 to err on the conservative side in the power calculation in case colonoscopy disutility was lower and sensitivity higher than expected.

\section{Discussion}

This study illustrates the application of a formal method to transform the overall harms and benefits of competing interventions into a measure of the non-inferiority margin. This method offers the benefits of allowing for more explicit and rigorous quantification of various considerations for determining that an intervention is non-inferior. It is applicable to all non-inferiority trials investigating an intervention that is expected slightly less effective as the standard intervention but is associated with less burden and side effects, either because of a de-escalation of the intervention or because of an alternative less invasive treatment regimen. The method may also be used for interventions that are slightly less effective but have (considerably) lower costs.

Our suggested approach for setting the non-inferiority margin is not substantially different from the Delphic method or the approach currently used by trialists who focus on disease outcomes alone. Both aim to set the margin such that the lower effectiveness is compensated by the lower burden, adverse effects, or resource requirements. The important difference between those approaches and the approach in this paper is the explication of all assumptions in our approach, and the consideration of lifetime effects. Using a decision model to estimate QALYs for the standard and alternative interventions ensures that the assumed trade-off between harms and benefits can be reproduced and the enduring impact of differences in interventions on quality of life can be incorporated.

There are several examples of successfully implemented Quality of Life (QoL) clinical trials, where non-inferiority margins are given in QALYs [23-27]. Our approach differs fundamentally from these trials in three ways. First, our approach uses QALYs to actually derive the non-inferiority margin, rather than just defining the non-inferiority margin in terms of QALYs. Second, these studies lack strict standards and a reproducible methodological framework. Finally, and most importantly, these studies suggest the establishment of a non-inferiority margin based on an acceptable loss in QALYs, whereas we suggest that the very definition of a non-inferiority margin requires equipoise in QALYs between the standard and the alternative treatment when the end-points are measured in terms of incidence or mortality outcomes.

This paper suggests an approach for setting a non-inferiority margin for non-inferiority trials, such that appropriate sample size can be estimated in a robust and reproducible way. These considerations for trial design should not be confused with Value of Information analyses which also use decision models [28]. These approaches, such as Expected Value of Sample Information, have been suggested to evaluate whether the resources needed to conduct a trial weigh up against the expected benefits of the additional information to be gained to advance medical decision making.

The impact of our proposed framework on the required sample size for non-inferiority trials depends on the trade-off between lower LY because of lower effectiveness and increase in quality-of-life because of reduced burden of the alternative intervention. In our example, we balance the small QALY gains by forgoing one colonoscopy for everyone versus the large QALY loss for the very few extra people diagnosed with CRC. This resulted in a relatively modest non-inferiority margin and a higher required sample size than currently used in the EPoS trial. However, for other examples of non-inferiority trials where the tradeoffs are much starker, such as a trial for local/regional HPV associated oropharynx cancer [29], the trade-offs may be much more pronounced. Here, the standard therapy is radiotherapy with cisplatin, while the alternative is radiotherapy with cetuximab. The primary end point is overall survival, under the assumption that a cetuximab-based radiotherapy will lead to less morbidity and better quality of life without a significant difference in overall survival or locoregional control. In this case, the increase in quality-of-life from the alternative intervention is very substantial and non-inferiority margin can be considerably wider. Accordingly, this will result in smaller required sample sizes.

In our example, we saw that a large reduction in the necessary sample size of the trial resulted from the incorporation of monetary costs in addition to adverse effects on health-related quality of life as the criterion for determining the non-inferiority margin. Boyd et al. [30] previously demonstrated the use of economic considerations to guide non-inferiority margins. Using cost-utility as an outcome requires defining an acceptable threshold for the cost of a loss in QALYs from the standard to the alternative intervention. Securing consensus on an appropriate cost-per-QALY threshold is fraught with difficulty, especially in the United States. Moreover, empiric data suggest that the threshold cost for forgoing health benefits, such as in the context of a non-inferiority trial, may in fact be higher than the price for gaining health benefits [31, 32]. However, it is important to recognize that medical interventions are not cost-neutral to society nor to patients themselves, who may experience direct financial toxicity from medical expenses, non-medical expenses, co-pays, loss of income, and other mechanisms. Therefore, it may be 
appropriate to consider whether such costs (at the societal or at least individual patient level) may be relevant to incorporate into clinical trial design.

The EPoS I example with the MISCAN-Colon model illustrates the feasibility of our suggested approach. It does not include establishment of effectiveness compared to a placebo control, as the FDA 95-95 and synthesis methods. However, the issue of ensuring that the non-inferiority margin does not overlap with the relapse rate of a placebo control can be an additional constraint after the size of the margin is determined by weighing the lower benefits of the alternative therapy against its putative lower morbidity burden.

In our case, we used an established sophisticated decision model which was accessible to us. While this model cannot be directly generalized to other situations, our proposed framework can be readily applied to other decision models and diseases. One could develop simpler decision models using Excel, R, or TreeAge, especially for treatment interventions, or use previously developed models and apply these within this framework [19, 33-35]. The most important requirements are case-by-case parameters on effectiveness and disutility of the standard intervention and on disutility of the alternative intervention. In these instances, there is value in trialists and modelers collaborating to formally apply this framework.

While some may argue that disutilities are inherently subjective, difficult to measure, and may have wide variability across the population, they are, by definition, an integral contributor to the size of the non-inferiority margin. The decision analytic framework approach proposed in this paper will allow trial designers to break down and understand the sensitivity of the non-inferiority margin to its component contributors (i.e. the chance of various events occurring and their associated disutilities), rather than using clinical ad hoc judgment to conjecture an estimate of this entire complex quantity at once.

Unfortunately, disutility estimates are often lacking like in our example. However, this framework would still allow postulation of a range of utilities and relate these to the non-inferiority margin and the associated trial sample size. Such an approach would make the implied disutilities of the alternative intervention explicit in the choice for the non-inferiority margin.

In the meantime, inclusion of patient-reported outcome measures that can be expressed and interpreted as a utility in early phase trials can provide pivotal data to help size later trials. However, there are a number of research challenges to be surmounted before population-level utility estimates required for a decision-analytic approach are available to justify sample size in non-inferiority trials. First, disutilities associated with acute effects of cancer screening or treatment have not been well studied, and this is particularly true of serious late treatment effects (e.g. cardiopulmonary toxicity and second malignancies) $[36,37]$. Second, point estimates represent the average utility for a population [38, 39], yet equally important is the variability in those utilities across the population and within specific subgroups [40-42], as well as at specific points in the treatment trajectory [43]. Third, although a growing number of health-related quality of life measures can be summarized and interpreted as both a score and a utility [44, 45], most measures of other constructs relevant to gauging the value of a cancer therapy (e.g. cost, treatment burden/complexity) $[46,47]$ do not yet have established utilities. Work to expand the range of patient-reported outcome measures [48-50] with associated utilities is warranted. Lastly, utilities are often seen as a corollary, rather than an essential component of a non-inferiority trial [51-55].

\section{Conclusions}

In sum, to maximize the rigor and efficiency of future clinical trials seeking to evaluate outcomes after de-escalation of (cancer) interventions, explicit quantification of benefits and harms of an intervention and its omission, along with the impact of each on quality of life, can be a particularly helpful approach. For example, interest has grown in recent years regarding the potential to omit adjuvant radiation therapy after breast conserving surgery for patients with early-stage breast cancer with favorable biologic features [56, 57]. Indeed, it was this particular issue that initially motivated the current exercise-as a means by which to refine the approach towards developing a feasible and sensible trial design in this context. In a trial evaluating such an option, the acceptable increase in breast cancer recurrence risk from omission of therapy can be estimated by weighing the disutility of excess cancer recurrence against the improvements in quality of life from avoidance of the intervention and its side effects. For such evaluations, it is important that trials are not censored at first events but continue to collect information on health outcomes, especially patient-reported quality of life measures and information on other events, after that.

In conclusion, we feel that those considering developing non-inferiority trials could consider innovative approaches to non-inferiority trial design such as the one we outline here, to design research that is simultaneously efficient, rigorous, and meaningful for patients facing complex cancer surveillance, prevention, and management decisions.

\section{Abbreviations}

CISNET: Cancer Intervention and Surveillance Modeling Network; CRC: Colorectal cancer; EPOS study: European Polyp Surveillance study; QALYs: Quality-adjusted life years; QoL: Quality of life 


\section{Acknowledgements}

Not applicable.

\section{Funding}

Financial support for this study was provided partly by a grant from the National Cancer Institute at the National Institutes of Health, through the Cancer Intervention and Surveillance Modeling Network (grant numbers U01-CA-152959, U01 CA199218). The research was also supported in part by a Lombardi Comprehensive Cancer Center American Cancer Society Young Investigator Award (ACS IRG 92-152-20 (PI Atkins) and the Cancer Prevention Research Fellowship sponsored by the American Society of Preventive Oncology and Breast Cancer Research Foundation (ASPO-17-001) to Dr. Jayasekera. The funding agreement ensured the authors' independence in designing the study, interpreting the data, writing, and publishing the report. The following authors are employed by the sponsor: EJF, SAM.

\section{Availability of data and materials}

Data sharing is not applicable to this article as no datasets were generated or analysed during the current study.

\section{Authors' contributions}

ILV participated in the design of the study, performed the statistical analysis, interpreted the results of the analysis and drafted the manuscript; RJ, JJ, NKS and SAM advised on the decision modeling and the statistical analysis, interpreted the results of the analysis, drafted the manuscript; EJF participated in the design of the study, interpreted the results of the analysis and drafted the manuscript. All authors read and approved the final manuscript.

\section{Ethics approval and consent to participate}

Not applicable.

\section{Consent for publication}

Not applicable.

\section{Competing interests}

The authors declare that they have no competing interests.

\section{Publisher's Note}

Springer Nature remains neutral with regard to jurisdictional claims in published maps and institutional affiliations.

\section{Author details \\ ${ }^{1}$ Department of Public Health, Erasmus MC University Medical Center Rotterdam, Rotterdam, the Netherlands. ${ }^{2}$ University of Michigan, Ann Arbor, MI, USA. ${ }^{3}$ Georgetown University Medical Center, Washington, DC, USA. ${ }^{4}$ Department of Population Medicine, Harvard Medical School and Harvard Pilgrim Health Care Institute, Boston, MA, USA. ${ }^{5}$ Healthcare Delivery Research Program, Division of Cancer Control and Population Sciences, National Cancer Institute, Bethesda, MD, USA. ${ }^{6}$ Statistical Research and Applications Branch, Surveillance Research Program, Division of Cancer Control and Population Sciences, National Cancer Institute, 9609 Medical Center Drive, Room 4E534, Bethesda, MD 20892-9765, USA.}

Received: 11 July 2018 Accepted: 13 December 2018 Published online: 07 January 2019

\section{References}

1. Walker E, Nowacki AS. Understanding equivalence and noninferiority testing. J Gen Intern Med. 2011;26(2):192-6.

2. The European Agency for the Evaluation of Medicinal Products - Committee for Proprietary Medicinal Products (2000). Points to consider on switching between superiority and non-inferiority. London, UK. http://www.ema. europa.eu/docs/en_GB/document_library/Scientific_guideline/2009/09/ WC500003658.pdf, last accessed on 12/20/2018.

3. European Medicines Agency - Committee for Medicinal Products for Human Use (2006). Guideline on the choice of the non-inferiority margin. London; UK. http://www.ema.europa.eu/docs/en_GB/document_library/ Scientific quideline/2009/09/WC500003636.pdf, last accessed on 12/20/ 2018.
4. International Council for Harmonisation of Technical Requirements for Pharmaceuticals for Human Use (2006). ICH topic E9 - Statistical Principles for Clinical Trials. Geneva, Switzerland. http://www.ich.org/fileadmin/Public_ Web_Site/ICH_Products/Guidelines/Efficacy/E9/Step4/E9_Guideline.pdf, last accessed on 12/20/2018.

5. International Council for Harmonisation of Technical Requirements for Pharmaceuticals for Human Use (2006). ICH Topic E10 - Choice of Control Group and Related Issues in Clinical Trials. Geneva, Switzerland. http://www. ich.org/fileadmin/Public_Web_Site/ICH_Products/Guidelines/Efficacy/E10/ Step4/E10_Guideline.pdf, last accessed on 12/20/2018.

6. Piaggio G, Elbourne DR, Pocock SJ, Evans SJ, Altman DG, Group C. Reporting of noninferiority and equivalence randomized trials: extension of the CONSORT 2010 statement. JAMA. 2012:308(24):2594-604.

7. Chan AW, Tetzlaff JM, Altman DG, et al. SPIRIT 2013 statement: defining standard protocol items for clinical trials. Ann Intern Med. 2013;158(3):200-7.

8. Chan AW, Tetzlaff JM, Gotzsche PC, et al. SPIRIT 2013 explanation and elaboration: guidance for protocols of clinical trials. BMJ. 2013;346:e7586.

9. Non-Inferiority Clinical Trials to Establish Effectiveness Guidance for Industry. U.S. Department of Health and Human Services, Food and Drug Administration, Center for Drug Evaluation and Research (CDER), Center for Biologics Evaluation and Research (CBER); 01/05/2016 November 2016.

10. Rehal S, Morris TP, Fielding K, Carpenter JR, Phillips PP. Non-inferiority trials: are they inferior? A systematic review of reporting in major medical journals. BMJ Open. 2016;6(10):e012594.

11. Torrance GW. Toward a utility theory foundation for health status index models. Health Serv Res. 1976;11(4):349-69.

12. Schumi J, Wittes JT. Through the looking glass: understanding noninferiority. Trials. 2011;12(1):106

13. Jover R, Bretthauer M, Dekker E, et al. Rationale and design of the European polyp surveillance (EPOS) trials. Endoscopy. 2016;48(6):571-8.

14. van Hees F, Zauber AG, van Veldhuizen $H$, et al. The value of models in informing resource allocation in colorectal cancer screening: the case of the Netherlands. Gut. 2015;64(12):1985-97.

15. van Hees F, Habbema JD, Meester RG, Lansdorp-Vogelaar I, van Ballegooijen M, Zauber AG. Should colorectal cancer screening be considered in elderly persons without previous screening? A cost-effectiveness analysis. Ann Intern Med. 2014;160(11):750-9.

16. Meester RG, Doubeni CA, Lansdorp-Vogelaar I, et al. Variation in adenoma detection rate and the lifetime benefits and cost of colorectal Cancer screening: a microsimulation model. JAMA. 2015; 313(23):2349-58

17. Knudsen AB, Zauber AG, Rutter CM, et al. Estimation of benefits, burden, and harms of colorectal Cancer screening strategies: modeling study for the US preventive services task force. JAMA. 2016;315(23):2595-609.

18. Rutter CM, Knudsen AB, Marsh TL, et al. Validation of models used to inform colorectal Cancer screening guidelines: accuracy and implications. Med Decis Mak. 2016;36(5):604-14.

19. Lansdorp-Vogelaar I, Gulati R, Mariotto AB, et al. Personalizing age of cancer screening cessation based on comorbid conditions: model estimates of harms and benefits. Ann Intern Med. 2014;161(2):104-12.

20. Loeve F, Boer R, van Oortmarssen GJ, van Ballegooijen M, Habbema JD. The MISCAN-COLON simulation model for the evaluation of colorectal cancer screening. Comput Biomed Res. 1999;32(1):13-33.

21. Wilschut JA, Hol L, Dekker E, et al. Cost-effectiveness analysis of a quantitative immunochemical test for colorectal cancer screening. Gastroenterology. 2011;141(5):1648-55 e1641.

22. Ness RM, Holmes AM, Klein R, Dittus R. Utility valuations for outcome states of colorectal cancer. Am J Gastroenterol. 1999;94(6):1650-7.

23. Langley RE, Stephens RJ, Nankivell M, et al. Interim data from the Medical Research Council QUARTZ trial: does whole brain radiotherapy affect the survival and quality of life of patients with brain metastases from non-small cell lung Cancer? Clin Oncol. 2013;25(3):e23-30.

24. Mulvenna PM, Nankivell MG, Barton $R$, et al. Whole brain radiotherapy for brain metastases from non-small lung cancer: Quality of life (QoL) and overall survival (OS) results from the UK Medical Research Council QUARTZ randomised clinical trial (ISRCTN 3826061). J Clin Oncol (Meeting Abstracts). 2015;33(15_suppl):8005.

25. Jobanputra P, Maggs F, Deeming A, et al. A randomised efficacy and discontinuation study of etanercept versus adalimumab (RED SEA) for rheumatoid arthritis: a pragmatic, unblinded, non-inferiority study of first TNF inhibitor use: outcomes over 2 years. BMJ Open. 2012;2(6):e001395. 
26. den Broeder AA, van Herwaarden N, van der Maas A, et al. Dose REduction strategy of subcutaneous TNF inhibitors in rheumatoid arthritis: design of a pragmatic randomised non inferiority trial, the DRESS study. BMC Musculoskelet Disord. 2013;14:299.

27. Collinson FJ, Gregory WM, McCabe C, et al. The STAR trial protocol: a randomised multi-stage phase I//II study of Sunitinib comparing temporary cessation with allowing continuation, at the time of maximal radiological response, in the first-line treatment of locally advanced/metastatic renal cancer. BMC Cancer. 2012;12:598.

28. Steuten L, van de Wetering G, Groothuis-Oudshoorn K, Retel V. A systematic and critical review of the evolving methods and applications of value of information in academia and practice. PharmacoEconomics. 2013;31(1):25-48.

29. Kofler B, Laban S, Busch CJ, Lorincz B, Knecht R. New treatment strategies for HPV-positive head and neck cancer. Eur Arch Otorhinolaryngol. 2014; 271(7):1861-7.

30. Boyd KA, Briggs AH, Fenwick E, Norrie J, Stock S. Power and sample size for cost-effectiveness analysis: fFN neonatal screening. Contemp Clin Trials. 2011;32(6):893-901.

31. Dowie J. Why cost-effectiveness should trump (clinical) effectiveness: the ethical economics of the south west quadrant. Health Econ. 2004;13(5):453-9.

32. O'Brien BJ, Gertsen K, Willan AR, Faulkner LA. Is there a kink in consumers' threshold value for cost-effectiveness in health care? Health Econ. 2002; $11(2): 175-80$

33. Canfell K, Chesson H, Kulasingam SL, Berkhof J, Diaz M, Kim JJ. Modeling preventative strategies against human papillomavirus-related disease in developed countries. Vaccine. 2012;30(Suppl 5):F157-67.

34. de Koning HJ, Meza R, Plevritis SK, et al. Benefits and harms of computed tomography lung cancer screening strategies: a comparative modeling study for the U.S. preventive services task force. Ann Intern Med. 2014; 160(5):311-20

35. Kroep S, Heberle CR, Curtius K, et al. Radiofrequency ablation of Barrett's esophagus reduces esophageal adenocarcinoma incidence and mortality in a comparative modeling analysis. Clin Gastroenterol Hepatol. 2017;15(9):1471-4.

36. Carlotto A, Hogsett VL, Maiorini EM, Razulis JG, Sonis ST. The economic burden of toxicities associated with cancer treatment: review of the literature and analysis of nausea and vomiting, diarrhoea, oral mucositis and fatigue. PharmacoEconomics. 2013;31(9):753-66.

37. Beusterien KM, Davies J, Leach M, et al. Population preference values for treatment outcomes in chronic lymphocytic leukaemia: a cross-sectional utility study. Health Qual Life Outcomes. 2010;8:50.

38. Krahn M, Ritvo P, Irvine J, et al. Patient and community preferences for outcomes in prostate cancer: implications for clinical policy. Med Care. 2003; 41(1):153-64.

39. Tong BC, Wallace S, Hartwig MG, D'Amico TA, Huber JC. Patient preferences in treatment choices for early-stage lung Cancer. Ann Thorac Surg. 2016; 102(6):1837-44.

40. Fu AZ, Graves KD, Jensen RE, Marshall JL, Formoso M, Potosky AL. Patient preference and decision-making for initiating metastatic colorectal cancer medical treatment. J Cancer Res Clin Oncol. 2016;142(3):699-706.

41. Nafees B, Lloyd AJ, Dewilde S, Rajan N, Lorenzo M. Health state utilities in non-small cell lung cancer: an international study. Asia Pac J Clin Oncol. 2017;13(5):e195-203.

42. Craig BM, Reeve BB, Cella D, Hays RD, Pickard AS, Revicki DA. Demographic differences in health preferences in the United States. Med Care. 2014;52(4): 307-13.

43. Schilling C, Dowsey MM, Clarke PM, Choong PF. Using patient-reported outcomes for economic evaluation: getting the timing right. Value Health. 2016;19(8):945-50.

44. Marriott ER, van Hazel G, Gibbs P, Hatswell AJ. Mapping EORTC-QLQ-C30 to EQ-5D-3L in patients with colorectal cancer. J Med Econ. 2017;20(2):193-9.

45. Hao Y, Wolfram V, Cook J. A structured review of health utility measures and elicitation in advanced/metastatic breast cancer. Clinicoecon Outcomes Res. 2016;8:293-303.

46. Eton DT, Yost KJ, Lai JS, et al. Development and validation of the patient experience with treatment and self-management (PETS): a patient-reported measure of treatment burden. Qual Life Res. 2017;26(2):489-503.

47. de Souza JA, Yap BJ, Wroblewski K, et al. Measuring financial toxicity as a clinically relevant patient-reported outcome: the validation of the COmprehensive Score for financial Toxicity (COST). Cancer. 2017;123(3):47684.
48. Craig BM, Mitchell SA. Examining the value of menopausal symptom relief among US women. Value Health. 2016;19(2):158-66

49. Lloyd AJ, Kerr C, Penton J, Knerer G. Health-related quality of life and health Utilities in Metastatic Castrate-Resistant Prostate Cancer: a survey capturing experiences from a diverse sample of UK patients. Value Health. 2015;18(8):1152-7.

50. Hays RD, Revicki DA, Feeny D, Fayers P, Spritzer KL, Cella D. Using linear equating to map PROMIS((R)) Global Health items and the PROMIS-29 V2.0 profile measure to the health utilities index mark 3. PharmacoEconomics. 2016;34(10):1015-22.

51. Xie J, Hao Y, Zhou ZY, Qi CZ, De G, Gluck S. Economic evaluations of Everolimus versus other hormonal therapies in the treatment of HR+/HER2advanced breast Cancer from a US payer perspective. Clin Breast Cancer. 2015;15(5):e263-76.

52. Yokomizo A, Kanimoto Y, Okamura T, et al. Randomized controlled study of the efficacy, safety and quality of life with low dose bacillus CalmetteGuerin instillation therapy for nonmuscle invasive bladder Cancer. J Urol. 2016;195(1):41-6.

53. Siu LL, Waldron JN, Chen BE, et al. Effect of standard radiotherapy with cisplatin vs accelerated radiotherapy with Panitumumab in locoregionally advanced squamous cell head and neck carcinoma: a randomized clinical trial. JAMA Oncol. 2017;3(2):220-6.

54. Tassinari D, Scarpi E, Sartori S, et al. Noninferiority trials in second-line treatments of nonsmall cell lung cancer: a systematic review of literature with meta-analysis of phase III randomized clinical trials. Am J Clin Oncol. 2012;35(6):593-9.

55. Amdahl J, Diaz J, Park J, Nakhaipour HR, Delea TE. Cost-effectiveness of pazopanib compared with sunitinib in metastatic renal cell carcinoma in Canada. Curr Oncol. 2016;23(4):e340-54.

56. Jagsi R. Early-stage breast cancer: falling risks and emerging options. Lancet. 2017;390(10099):1010-1012

57. Liu FF, Shi W, Done SJ, et al. Identification of a low-risk luminal a breast Cancer cohort that may not benefit from breast radiotherapy. J Clin Oncol. 2015;33(18):2035-40.
Ready to submit your research? Choose BMC and benefit from:

- fast, convenient online submission

- thorough peer review by experienced researchers in your field

- rapid publication on acceptance

- support for research data, including large and complex data types

- gold Open Access which fosters wider collaboration and increased citations

- maximum visibility for your research: over $100 \mathrm{M}$ website views per year

At BMC, research is always in progress.

Learn more biomedcentral.com/submissions 